\title{
Inhibitory Effect of Endostar on Specific Angiogenesis Induced by Human Hepatocellular Carcinoma
}

\author{
Qing Ye, ${ }^{1,2,3}$ Shukui Qin, ${ }^{1,2}$ Yanhong Liu, ${ }^{3}$ Jundong Feng, ${ }^{1,2}$ Qiong Wu, \\ Wenshu $Q u^{2}{ }^{2}$ and Xiaojin Yin ${ }^{4}$ \\ ${ }^{1}$ Postdoctoral Station of Nanjing General Hospital, Nanjing 210002, China \\ ${ }^{2}$ PLA Cancer Center in Eighty-One Hospital of People's Liberation Army, Nanjing 210002, China \\ ${ }^{3}$ Department of Pathology, Affiliated Drum Tower Hospital of Nanjing University Medical School, Nanjing 210008, China \\ ${ }^{4}$ Jiangsu Harbinger Drug Development Co., Ltd., Nanjing 210002, China
}

Correspondence should be addressed to Shukui Qin; qinsk@csco.org.cn and Xiaojin Yin; 316004095@qq.com

Received 25 August 2014; Accepted 10 December 2014

Academic Editor: Zhongxia Wang

Copyright (C) 2015 Qing Ye et al. This is an open access article distributed under the Creative Commons Attribution License, which permits unrestricted use, distribution, and reproduction in any medium, provided the original work is properly cited.

\begin{abstract}
To investigate the effect of endostar on specific angiogenesis induced by human hepatocellular carcinoma, this research systematically elucidated the inhibitory effect on HepG2-induced angiogenesis by endostar from $50 \mathrm{ng} / \mathrm{mL}$ to $50000 \mathrm{ng} / \mathrm{mL}$. We employed fluorescence quantitative Boyden chamber analysis, wound-healing assay, flow cytometry examination using a coculture system, quantitative analysis of tube formation, and in vivo Matrigel plug assay induced by HCC conditioned media (HCM) and HepG2 compared with normal hepatocyte conditioned media (NCM) and L02. Then, we found that endostar as a tumor angiogenesis inhibitor could potently inhibit human umbilical vein endothelial cell (HUVEC) migration in response to HCM after four- to six-hour action, inhibit HCM-induced HUVEC migration to the lesion part in a dose-dependent manner between $50 \mathrm{ng} / \mathrm{mL}$ and $5000 \mathrm{ng} / \mathrm{mL}$ at 24 hours, and reduce HUVEC proliferation in a dose-dependent fashion. Endostar inhibited HepG2induced tube formation of HUVECs which peaked at $50 \mathrm{ng} / \mathrm{mL}$. In vivo Matrigel plug formation was also significantly reduced by endostar in HepG2 inducing system rather than in L02 inducing system. It could be concluded that, at cell level, endostar inhibited the angiogenesis-related biological behaviors of HUVEC in response to HCC, including migration, adhesion proliferation, and tube formation. At animal level, endostar inhibited the angiogenesis in response to HCC in Matrigel matrix.
\end{abstract}

\section{Introduction}

Hepatocellular carcinoma (HCC) is the fifth most common tumor in the world, which is difficult to be cured and easily relapses. Surgical resection, liver transplantation, local interventional therapy, and general chemotherapy were conventional treatments with limitations $[1,2]$. It was imperative to find a new systematic therapy for HCC. General chemotherapeutics are aimed at tumor cells, while antiangiogenesis therapeutics are aimed at vascular endothelial cells which have become the second important target because of their stable performance, specificity, and wide scope $[3,4]$. HCC is a hyper vascularized tumor, where during it growth cancer cells induce angiogenesis through various pathways.

Abundant blood vessel was observed in HCC angiography, and angiogenesis was closely connected to HCC prognosis [5]. Immunological histological chemistry (IHC) using specific labeled CD34 in vascular endothelial cell to detect microvessel density of HCC was an extensively applied pathological indicator for HCC prognosis [6]. Successful antiangiogenesis therapy in colorectal cancer [7] and lung cancer $[8,9]$ provides new orientation for HCC antiangiogenesis therapy.

Endothelial cells play important roles in angiogenesis. Among multiple antiangiogenesis factors, endostatin was satisfactory because it is endogenous and multitargeted. Endostatin is the C-terminal amino fragment of type X VIII collagen, which was firstly separated from angioblastoma strains of mouse. Endostatin specifically inhibits proliferation of vascular endothelial cells and capillary growth [10]. It was reported that endostatin could induce cell apoptosis and cell cycle arrest of endothelial cells [11]. 
Animal experiments showed that endostatin could significantly repress the proliferation of various murine and allogeneic transplantation tumors [12]. However, there were some difficulties in the application of genetic engineering restructuring human endostatin in clinic because restructured endostatin expressed in E. coli was in form of inclusion body which was difficult to be purified and refolded [13]. Compared to endostatin, endostar was a newly restructured human vascular endothelial inhibitor with a His tag (MGGSHH$\mathrm{HHH}$ ) added to its $\mathrm{N}$-terminal which makes it to be purified easily [14]. However, whether this modification influences the biological activity of Endostatin needs further verification.

Some reports and statistics showed that endostar has potential effect on HCC therapy: (a) the density of microvessel in HCC lumps was a prognostic indicator of HCC relapse after surgery [15] and (b) the low expression level of endostatin in HCC and abundant angiogenesis were related to tumor progression [16]. (c) Endostar combined with navelbine and cisplatin in the therapy of another rich vascular tumor, NSCLC, showed that they could improve advanced stage therapy effect and prolong median time of progress of tumors in phases I, II, and III trial [17]. (d) Endostatin containing plasmid showed a suppression inhibitory effect towards human HCC cell line Bel-7402 and its heterogeneous transplantation tumor in nude mice model. (e) Novel angiogenesis inhibitor Avastin combining chemotherapy was in phase II clinic experiments which could prolong the progression free survival (PFS) of HCC patient for 6 months [18].

In this research, we employed HUVEC as the cell model and normal hepatocyte line L02. L02 was used as control with an antiangiogenesis evaluating in vitro and in vivo experiment system to study the effects of recombinant human endostatin (endostar) on human HCC cell line HepG2-induced angiogenesis-related biological behaviors of HUVECs, which could provide theoretical and experimental evidence for endostar being used as an antiangiogenesis drug in HCC treatment.

\section{Materials and Methods}

2.1. Cell Culture. Experiments were performed using HUVECs (ScienCell), HepG2 (from Shanghai Cell Institution), and L02 (from Shanghai Cell Institution). HUVECs were cultured in endothelial cell medium (ECM; ScienCell) supplemented with $1 \%$ endothelial cell growth supplement (ECGS; ScienCell), 5\% fetal bovine serum (FBS, Invitrogen Corp.), $100 \mathrm{U} / \mathrm{mL}$ penicillin (Invitrogen Corp.), and $100 \mu \mathrm{g} /$ $\mathrm{mL}$ streptomycin (Invitrogen Corp.). HepG2 and L02 were cultured in Dulbecco's modified eagle medium (DMEM, Invitrogen Corp.) supplemented with $10 \%$ FBS, $100 \mathrm{U} / \mathrm{mL}$ penicillin, and $100 \mu \mathrm{g} / \mathrm{mL}$ streptomycin. All the cells were maintained within the $5 \% \mathrm{CO}_{2}$ air atmosphere in a humidified incubator at $37^{\circ} \mathrm{C} .90 \%$ confluent cells were detached by trypsin/EDTA (Invitrogen Corp.) and were subcultured at proper split ratio.

2.2. Animals. BALB c/J-severe combined immunodeficient (SCID) mice were obtained from Shanghai SLAC Laboratory and were used for matrigel plug study.
2.3. Preparation of HCM from HepG2 and NCM from L02. As the method described by Moroz et al. [19], cells were allowed to grow till $80 \%$ confluence. Following the replacement of the culture medium with $15 \mathrm{~mL}$ DMEM plus $0.2 \%$ bovine serum albumin (BSA, Invitrogen Corp.), cells were placed within the $5 \% \mathrm{CO}_{2}$ air atmosphere in a humidified incubator at $37^{\circ} \mathrm{C}$ for $24 \mathrm{~h}$. HCM or NCM were collected and centrifuged for $15 \mathrm{~min}$ at 7,000 rpm to eliminate cell debris. The supernatants were ultra-filtered by Amicon Ultra-15 (Millipore, MWCO $3 \mathrm{KD}$ ) to obtain protein and were then resuspended in human endothelial serum-free medium (SFM; Invitrogen Corp.) plus $0.2 \%$ BSA. The suspension was collected and filtered. The collected medium was stored at $-80^{\circ} \mathrm{C}$.

2.4. Migration Assay. The HUVEC migration assays were performed as described in the protocol using 24-well modified Boyden chambers containing polyethylene membranes (Greiner Bio-One). HUVECs were starved overnight in SFM with $0.2 \%$ BSA. The cells were trypsinized, resuspended in SFM containing $0.2 \%$ BSA, and mixed with endostar (Simcere Pharmaceutical Co., Ltd.) of different final concentrations $(0 \mathrm{ng} / \mathrm{mL}, 5 \mathrm{ng} / \mathrm{mL}, 50 \mathrm{ng} / \mathrm{m}, 500 \mathrm{ng} / \mathrm{mL}, 5,000 \mathrm{ng} / \mathrm{mL}$, and $50,000 \mathrm{ng} / \mathrm{mL}$ ) for $30 \mathrm{~min}$ before adding the mixture to the upper chamber. HCM and NCM mixed with endostar served as attractants that were placed in the lower wells. SFM with $10 \%$ FBS and SFM with $0.2 \%$ BSA were employed as positive and negative controls, respectively. The cell culture plates were incubated in a cell culture incubator at $37^{\circ} \mathrm{C}$ and $5 \%$ $\mathrm{CO}_{2}$ for $6 \mathrm{~h}$. Subsequently, all cells were fluorescently labeled with $4 \mu \mathrm{M}$ calcein-AM (Sigma) for $45 \mathrm{~min}$ and incubated in prewarmed trypsin-EDTA for $10 \mathrm{~min}$, allowing migratory cells to detach from the underside of the PET membrane. Finally, the migratory cells were quantified in the TECAN multifunctional reader at an excitation wavelength of $485 \mathrm{~nm}$ and an emission wavelength of $520 \mathrm{~nm}$.

2.5. Wound-Healing Assay. Wound-healing assay was performed as previously described [20] with some improvements. HUVECs $\left(1.8 \times 10^{4}\right.$ per well $)$ were seeded in 24 well plates and cultured in ECM medium to $90 \%$ confluence. HUVECs were starved for $4 \mathrm{~h}$ in SFM with $0.2 \%$ BSA. The cell monolayer was scraped with a sterile tip to create a cellfree zone and then photographed to determine the injury baseline. Media were replaced by HCM containing endostar of different concentration. SFM with 5\% FBS and SFM with $0.2 \%$ BSA were used as controls. The migration of the cells was recorded at 24 hours on an Olympus IX-71 inverted microscope equipped with an Olympus camera. Data were analyzed by Image-Pro Plus software.

2.6. Cell Adhesion Assay. Adhesion between tumor cells and endothelial cells was measured as described with some modifications [21]. HepG2 and L02 were seeded in 96well plate to $100 \%$ confluence before experiments. HUVECs were stained with $4 \mu \mathrm{M}$ calcein-AM and trypsinized. Single HUVEC suspension was pretreated with endostar of different concentration for $30 \mathrm{~min}$. HUVECs were mixed with HCM before being seeded in the HepG2 cell-coating plate 
$\left(4 \times 10^{4}\right.$ per well). Plates were incubated at $37^{\circ} \mathrm{C}$ for $1 \mathrm{~h}$. To remove nonadherent cells, each well was carefully washed by addition of prewarmed serum-free culture medium, followed by gentle swirling and inversion of the plate and blotting of excess liquid onto filter paper or paper towels. This was repeated four times, after which $1 \mathrm{~mL}$ of prewarmed serumfree culture medium was added to each well. Relative fluorescence units (RFU) were measured using TECAN multifunctional reader at an excitation wavelength of $485 \mathrm{~nm}$ and an emission wavelength of $520 \mathrm{~nm}$. Adhesion rates were calculated by the formula: Adhesion rate $=($ RFU100\%control RFU0\%control)/100 × (RFUsample - RFU0\%control).

2.7. CFSE-Labeled Flow Cytometric Analysis. The coculture system was established as previously described [22] with some changes. Immediately before the assay, HUVECs and HepG2 were stained with carboxyfluorescein succinimidyl amino ester (CFSE) (Molecular Probes) and PKH-26 red fluorescent cell linker (Sigma-Aldrich), respectively, as the protocol. Parts of the labeled cells were fixed immediately and used as positive control. HUVECs were plated in 12-well plates $\left(4.2 \times 10^{4}\right.$ per well) alone or at ratio of 1:4 compared to HepG2. After complete attachment, cells were starved in SFM plus $0.2 \%$ BSA for $6 \mathrm{~h}$ and treated with endostar for an additional $1 \mathrm{~h}$. Media were replaced by mixture of SFM plus 2\% FBS together with endostar of corresponding concentrations. $48 \mathrm{~h}$ later, HUVECs were harvested, fixed in cell fixation liquid, and analyzed by flow cytometry. Data were analyzed by CELLQuest and ModFit to obtain proliferation index. Positive control was employed as the first generation. Inhibition rates were calculated by the following formula: $\mathrm{IR}=\left(\mathrm{PI}_{\mathrm{nc}}-\mathrm{PI}_{\text {sample }}\right) / \mathrm{PI}_{\mathrm{NC}} \times 100 \%$.

2.8. Tube Formation Assay. Tube formation was evaluated as previously described [20]. $50 \mu \mathrm{L}$ of diluted growth factorreduced Matrigel (Becton Dickinson) was tiled on the bottom of 96-well plates at $4^{\circ} \mathrm{C}$ and left at $37^{\circ} \mathrm{C}$ for gelification. HUVECs were seeded at a density of $2.25 \times 10^{4}$ per well in SFM, HCM, and NCM. For coculture experiments, mixtures of HUVECs and HepG2 at a 1:1 ratio were seeded on Matrigel in HCM. Endostar of different concentrations was added. All tube formation experiments were observed using Olympus IX-71 inverted fluorescence microscopy and images were digitally captured at 24 hours after plating. Tubule formation was assessed by counting the number of tubule branches and the total area covered by tubules in each field of view by Image-Pro Plus software.

2.9. In Vivo Matrigel Plug Assay. Angiogenesis was analyzed using the in vivo Matrigel plug assay as previously described [23]. Briefly, GFR-Matrigel, HepG2/L02, and endostar at a $2: 1: 1$ volume ratio were thoroughly mixed at $4^{\circ} \mathrm{C}$. Male $\mathrm{BALB} / \mathrm{c}$ SCID mice were anesthetized and injected with the mixture of GFR-Matrigel and HepG2 subcutaneously (s.c.) on the upper left dorsal. GFR-Matrigel and L02 cell mixture was injected on the upper right part using a prechilled tuberculin syringe (27-gauge needle). The mixture of GFRMatrigel and endostar was employed as blank control and

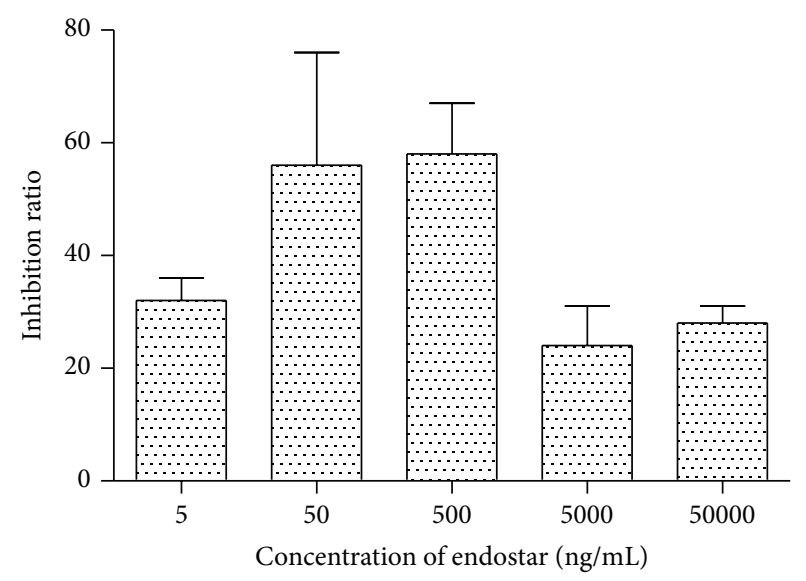

FIGURE 1: Effect of endostar towards HUVEC chemotaxis induced by HCM.

was injected on the bottom left dorsal. Starting from the second day, $8 \mathrm{mg} / \mathrm{kg}$ endostar was given to mice every day by intraperitoneal injection. Seven days after implantation, mice were sacrificed. Matrigel plugs with the surrounding skin were removed and vascularity was photographed (37). Then Matrigel plugs were homogenized in $500 \mu \mathrm{L}$ of radioimmunoprecipitation (RIPA; Beyotime) buffer. The homogenate was centrifuged at $1,000 \times \mathrm{g}$ to collect the suspension. OD values of Hemoglobin were measured at the absorbance wavelength $420 \mathrm{~nm}$ using a TECAN multifunctional reader. Results were analyzed by ANVOA test.

\section{Result}

3.1. The Effects of Endostar on HCC-Induced HUVEC Migration. Boyden chambers migration assay showed that when using HCM as attractants, migration of HUVEC was obviously induced in $4-6 \mathrm{~h}$ which could be inhibited by endostar with concentration of $5 \mathrm{ng} / \mathrm{mL}$ to $50,000 \mathrm{ng} / \mathrm{mL}$. When the concentration of endostar was $50 \mathrm{ng} / \mathrm{mL}$ and $500 \mathrm{ng} / \mathrm{mL}$, the suppression effect was most significant $(P<0.01)$ (Figure 1).

Wound-healing assay showed that HCM induced migration of HUVEC to wound area which could be suppressed by endostar (Figure 2(a)). After $24 \mathrm{~h}$, the suppression effect of endostar was concentration-dependent from the concentration of $50 \mathrm{ng} / \mathrm{mL}$ to $5,000 \mathrm{ng} / \mathrm{mL}$ (Figure 2(b)).

3.2. The Effects of Endostar on HCC-Induced HUVEC Adhesion. In cell adhesion assay, adhesion rate and suppression rate of each group were calculated by RFU as shown in Figure 3. It was revealed that the cell number of HUVEC that adhered to HepG2 was decreased by addition of endostar. The suppression effect of endostar towards adhesion ability of HUVEC to HepG2 increased as its concentration elevated from $50 \mathrm{ng} / \mathrm{mL}$ to $5,000 \mathrm{ng} / \mathrm{mL}$.

3.3. The Effects of Endostar on HCC-Induced HUVEC Proliferation. The result of CFSE-labeled flow cytometric analysis was analyzed by CELLQuest and ModFit and the PI value 

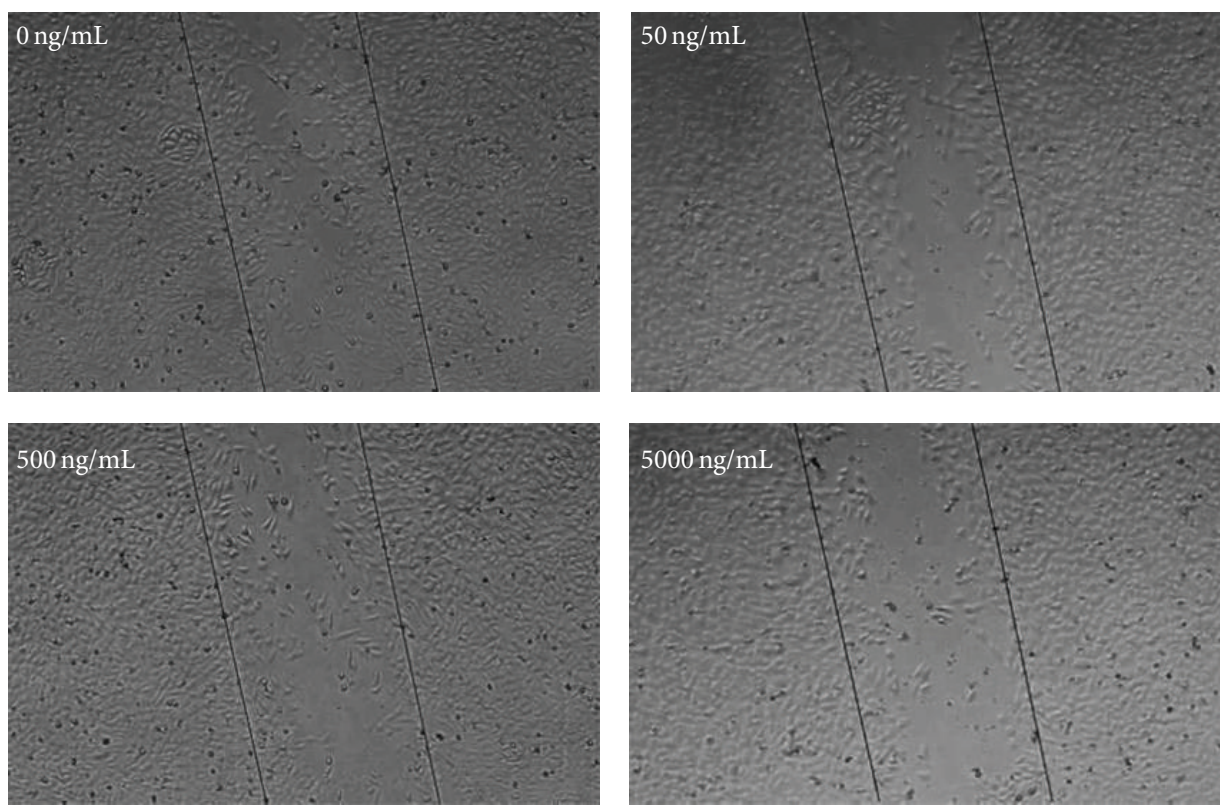

(a)

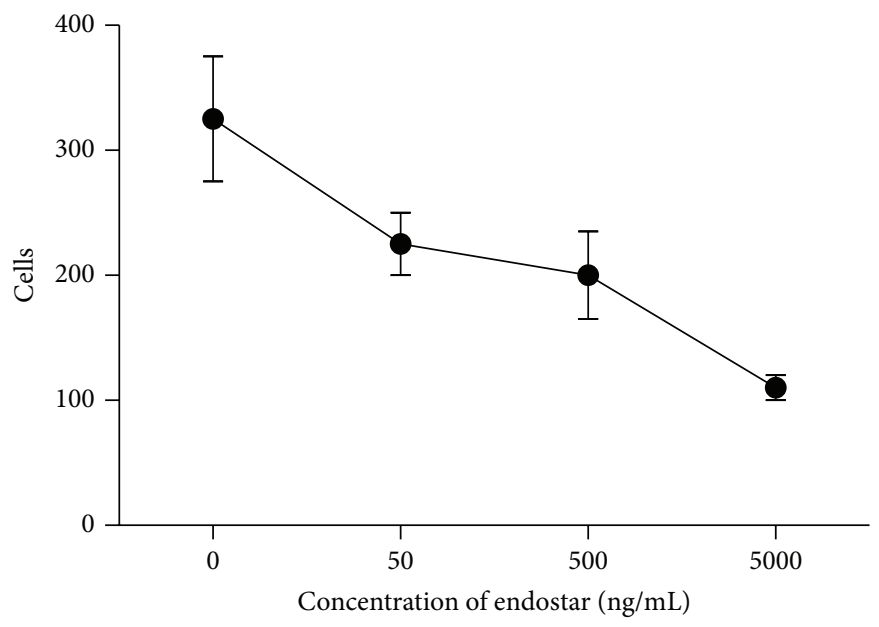

(b)

FIGURE 2: HCM induced migration of HUVEC to wound area was suppressed by endostar. (a) Endostar with different concentrations suppressed HUVEC migration detected by microscopy. (b) The amount of HUVEC cells migration suppressed by different concentrations of endostar.

of each sample was calculated and exhibited in Figure 4, which suggested that endostar significantly suppressed the proliferation of HUVEC in HepG2 cocultured system and HCM culture system dose-dependently. Furthermore, the suppression effect of endostar towards the proliferation of HUVEC was more significant in coculture system than in HCM system $(P<0.05)$.

3.4. The Effects of Endostar on HCC-Induced Tube Formation. Tube formation assay showed that the HUVEC net tube structure induced by HepG2 was significantly decreased by addition of endostar with different concentrations (Figure 5(a)). Analysis with Image-Pro Plus showed that the length (Figure 5(b)), area (Figure 5(c)), and amount (Figure 5(d)) of Matrigel net tube formed by HUVEC all decreased after endostar administration. The suppression effect was most significant when the concentration was $50 \mathrm{ng} / \mathrm{mL}$.

3.5. The Effects of Endostar on HCC-Induced Angiogenesis In Vivo. In vivo Matrigel plug assay showed that GFR-Matrigel + HepG2, GFR-Matrigel + L02 could form plug containing initial vessel in subcutaneous SCID mouse. The number of initial vessels in the plug was decreased by endostar. In Figure 6(a), when the concentration of endostar was $0 \mathrm{ng} / \mathrm{mL}$, there were a large number of initial vessels in the plug formed by GFR-Matrigel + HepG2 and the color of the plug was dark red. When the concentration of endostar was $50 \mathrm{ng} / \mathrm{mL}$, the initial vessels were significantly decreased 

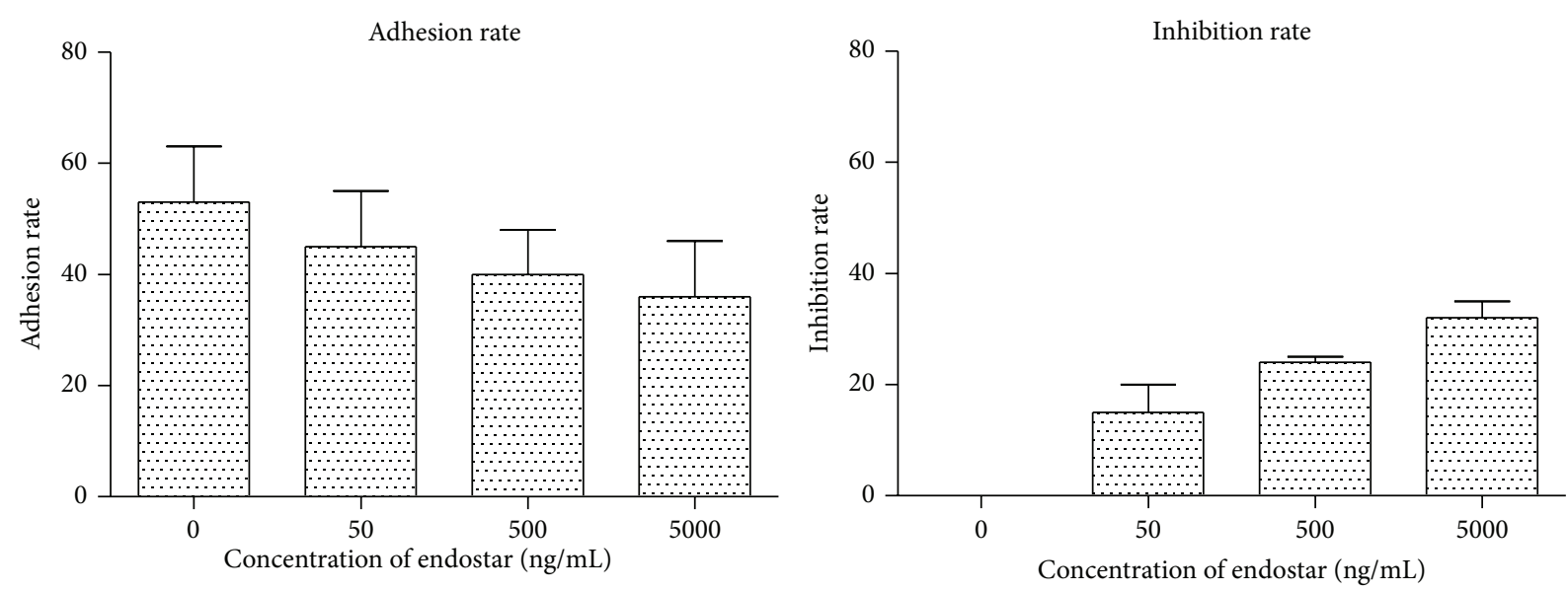

FIGURE 3: Adhesion rate and suppression rate effect by different endostar concentrations in cell adhesion assay.

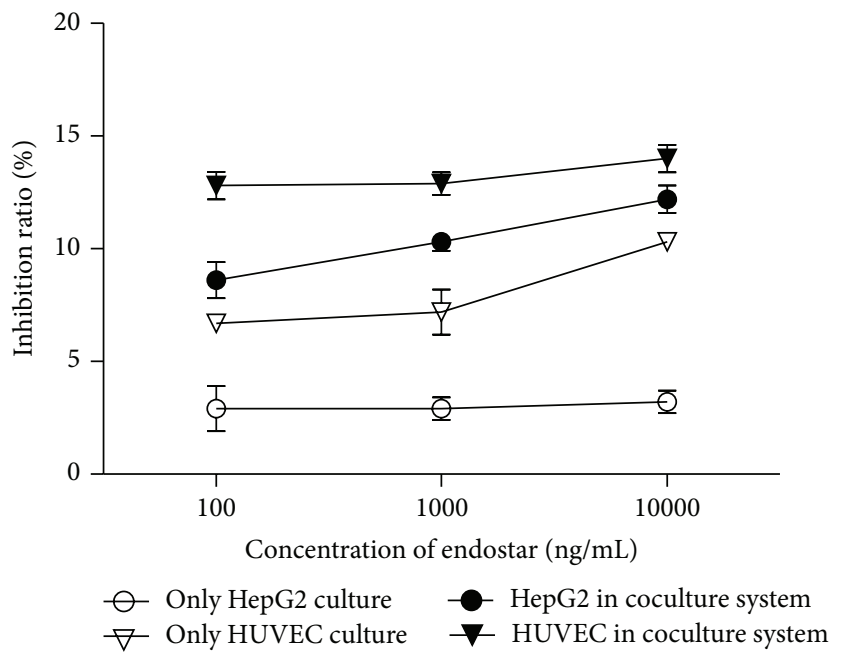

FIGURE 4: The effects of different concentrations of endostar on HCC-induced HUVEC proliferation detected by CFSE-labeled flow cytometric analysis.

except a huge initial vessel in the plug. When the concentration of endostar was $500 \mathrm{ng} / \mathrm{mL}$, there were some tiny vessels left in the plug. When the concentration of endostar was $5,000 \mathrm{ng} / \mathrm{mL}$, the color of plug was pale. After homogenization, the homogenate OD values of endostar-treated plugs decreased which suggested that endostar significantly suppressed HepG2-induced angiogenesis (Figure 6(b)).

\section{Discussion}

The mechanism of HCC-induced endothelial cell migration was generally considered to be related to various cytokines of HCC cells such as vascular endothelial growth factor (VEGF) family, fibroblast growth factors (FGF) family, Angiopoietin2, Angiotropin, tumor necrosis factor (TNF)- $\alpha$, colony stimulating factors (CSFs), CXC chemokines with ELR motif, hepatocyte growth factor (HGF), platelet endothelial cell adhesion molecule- (PECAM-) 1, and Integrins [24]. A network was established by interaction of these cytokines; however, the weight of each cytokines was unknown. In general, endostar could inhibit chemotactic migration of endothelial cells induced by the multiple cytokines secreted by HCC cells. In fluorescence quantitative migration chamber experiments, multiple cytokines induced chemotactic migration of endothelial cells, which was inhibited after endostar was added. When endostar was added for $4 \mathrm{~h}$ to $6 \mathrm{~h}$ with concentrations from $5 \mathrm{ng} / \mathrm{mL}$ to $50,000 \mathrm{ng} / \mathrm{mL}$, inhibitory effect was expressed and it was most significant with concentrations of $50 \mathrm{ng} / \mathrm{mL}$ and $500 \mathrm{ng} / \mathrm{mL}$.

Similar results were detected in scratch experiment. After scratch, HCM induced chemotactic migration of endothelial cells to injured part. When endostar was added for $24 \mathrm{~h}$, with the concentrations from $5 \mathrm{ng} / \mathrm{mL}$ to $50,000 \mathrm{ng} / \mathrm{mL}$, the inhibition of chemotactic migration ability of endothelial cells to injured part by HCM was concentration-dependent. Although the two experiments suggested that endostar could inhibit endothelial cell migration induced by HCM, the most effective concentration was different, which was dependent on different action time of endostar.

The adhesion mechanism of endothelial cell induced by HCC cells was generally considered to be related to the secretion of VEGF, FGF- 2 by HCC cells, and integrins $(\alpha 1 \beta 1$ and $\alpha 2 \beta 1$ ), VE-cadherin, and PECAM-1 upregulated in endothelial cells, which could improve the adhesion effect of endothelial cells to HCC cells [25]. Endostar can effectively suppress the adhesion of endothelial cell induced by HCC, which was detected by the experiment of fluorescent quantitative adhesion. The number of HUVEC cells that were activated by HCM and that adhered to HepG2 significantly decreased when endostar was added. When the concentration of endostar was from $5 \mathrm{ng} / \mathrm{mL}$ to $50,000 \mathrm{ng} / \mathrm{mL}$, the adhesion suppression effect was concentration-dependent.

HCC cells induce the proliferation of vessel endothelial cell via two approaches: one was secreting some cytokines such as VEGF-family, FGF-family, angiopoietin-2, EGF, CSFs, angiogenin, CXC chemokines with ELR motif, insulinlike growth factor- (IGF-) 1, erythropoietin, and interleukin(IL-) 8. Another was direct contact with vessel endothelial through integrins and VE-cadherin [26]. Coculture experiment showed that endostar significantly suppressed 

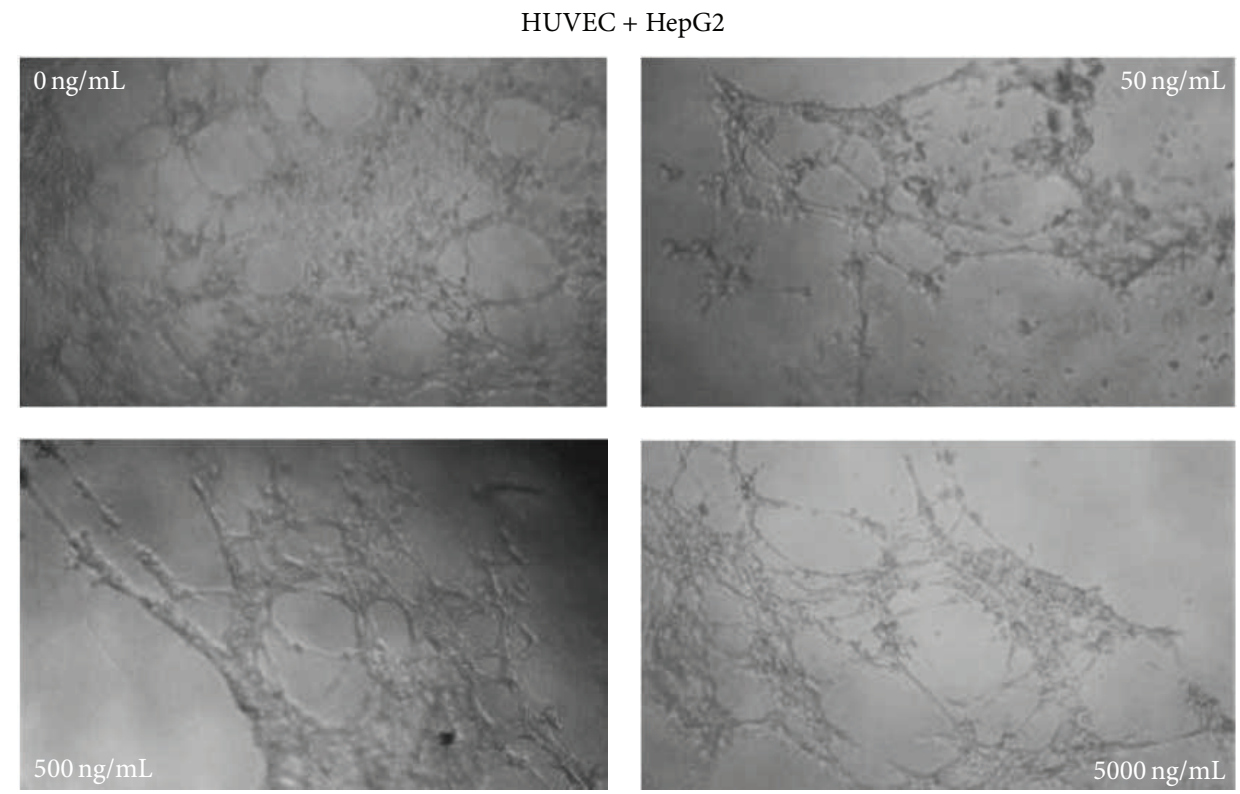

(a)

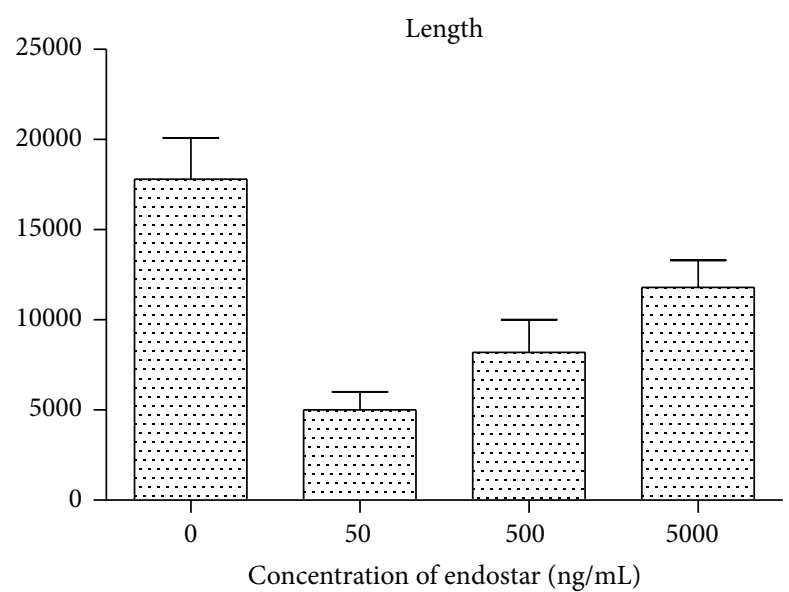

(b)

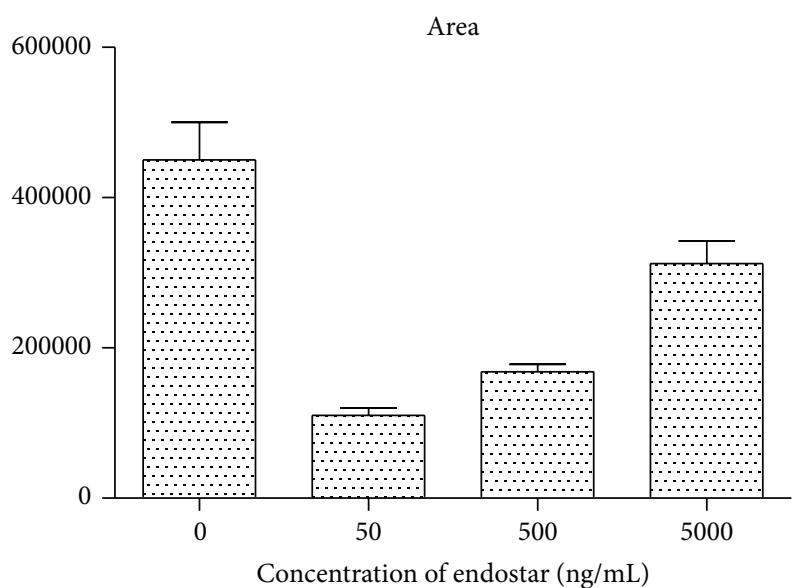

(c)

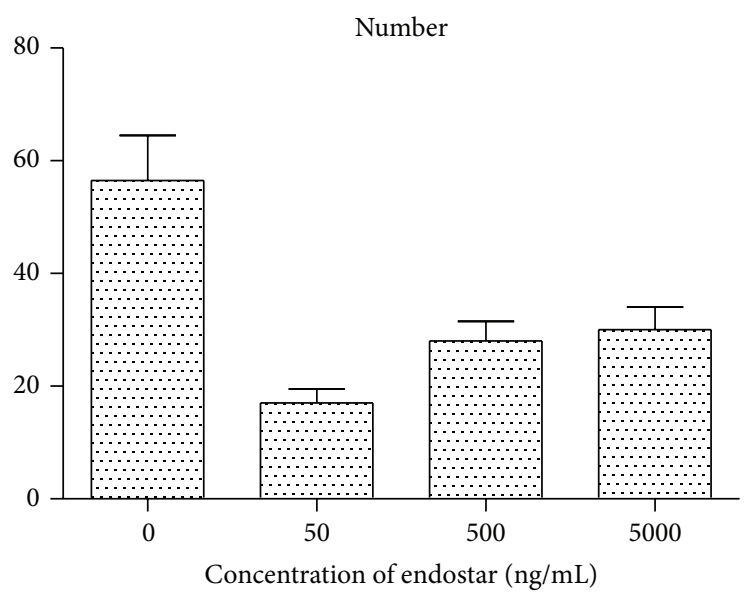

(d)

FIGURE 5: Tube formation of HUVEC induced by HCM was suppressed by endostar. (a) Endostar suppressed tube formation of HUVEC induced by HCM detected by microscopy. (b) Endostar suppressed the length of formatted net tube. (c) Endostar suppressed the area of formatted net tube. (d) Endostar suppressed the amount of formatted net tube. 


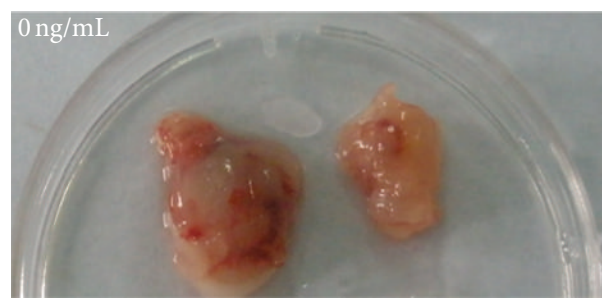

GFR-Matrigel + HepG2

GFR-Matrigel + L02

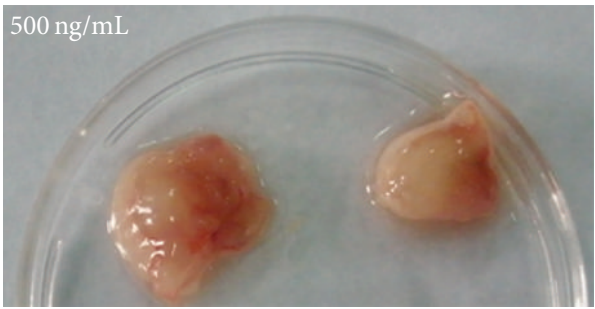

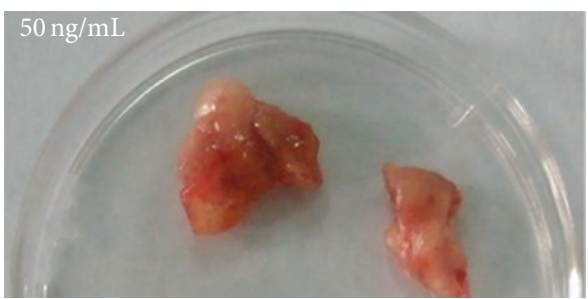

GFR-Matrigel + HepG2

GFR-Matrigel + L02

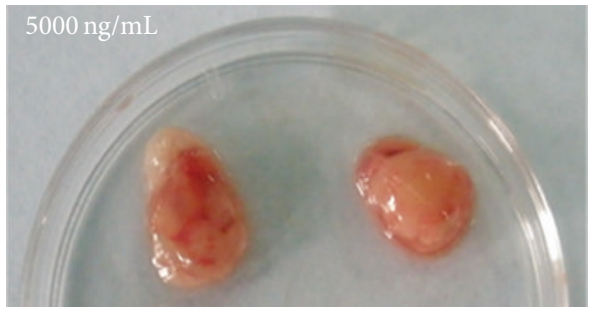

(a)

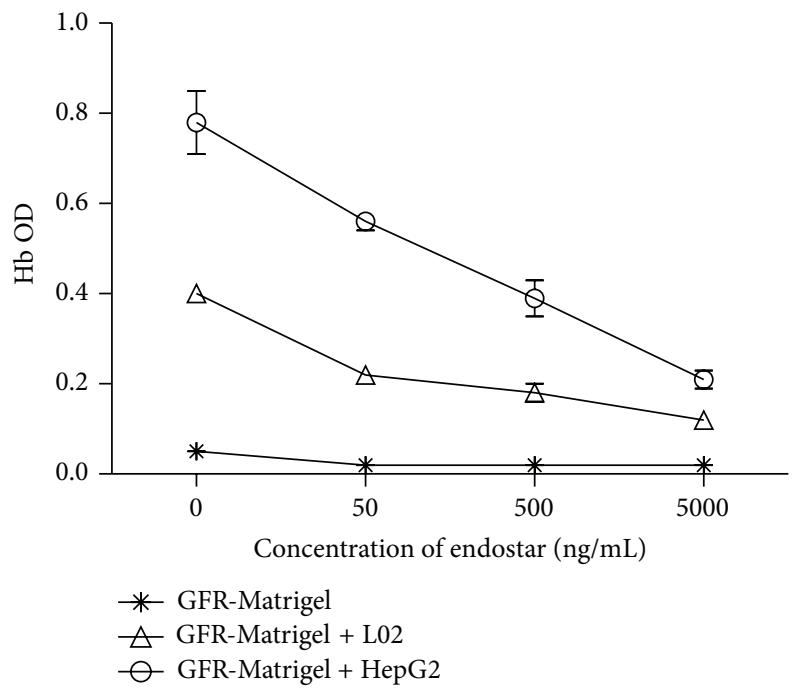

(b)

Figure 6: The suppression effect of endostar towards HCC-induced angiogenesis detected by in vivo Matrigel plug assay. (a) The Matrigel plugs formatted in SCID mouse. (b) The quantity of homogenate in Matrigel plugs.

the proliferation of HUVEC cocultured with HepG2 and cultured in HCM alone. The suppression effective was more obvious in HUVEC cocultured with HepG2 than in HUVEC cultured in HCM alone, which suggested that endostar suppressed the proliferation of endothelial cell affected by multiple factors and induced by HCC cells.

Similar results were detected in tube formation assay. In one way, HCC cells improved the formation of tubes by secreting active factors such as TGF- $\beta$, TNF- $\alpha$, and angiotropin [27]. In another way, HCC cells improved the formation of tubes via direct contact with PECAM-1 and VEcadherin [28].

In Matrigel, the net tube structure was obviously decreased by the addition of endostar. When the concentration of endostar was between $50 \mathrm{ng} / \mathrm{mL}$ and $5000 \mathrm{ng} / \mathrm{mL}$, the peak point of suppression was $50 \mathrm{ng} / \mathrm{mL}$, which suggested endostar suppressed the tube structure formation of endothelial cell affected by multiple factors and induced by HCC cells.

Multiple cytokines such as VEGF-family, TNF $\alpha$, EGF, angiotropin, and HGF were closely related to in vivo angiogenesis [29]. Our research results also showed that HCC cells could induce more blood vessels, while endostar significantly suppressed the induced angiogenesis. Detection of hemoglobin quantity in plugs showed that during the concentrations from $50 \mathrm{ng} / \mathrm{mL}$ to $5000 \mathrm{ng} / \mathrm{mL}$, endostar could significantly suppress HCC induced angiogenesis dose dependently.

In summary, our study using a series modified classical in vitro experiments such as Boyden chambers migration array, wound-healing assay, cell adhesion assay, CFSE-labeled flow cytometric analysis, and tube formation assay analyzed the suppression effect of endostar on HCC-induced endothelial 
cell migration, adhesion, proliferation, and tube formation, which suggest that endostar could suppress the angiogenesis of HCC in vitro. We also analyzed the suppression effect of HCC-induced angiogenesis in vivo by modified Matrigel plug assay suppress, which showed that endostar could suppress the angiogenesis of HCC in vivo.

The molecular mechanism of endostar suppressing HCCinduced angiogenesis still needs further elucidation. However, our research confirmed that endostar had suppression effect towards multiple factor effect and HCC-induced angiogenesis. The results provide theoretical and experimental proofs for the role of endostar in antiangiogenesis therapy.

\section{Conflict of Interests}

The authors declare that there is no conflict of interests regarding the publication of this paper.

\section{References}

[1] S. Zhong, "China's expert consensus on laparoscopic liver resection and guidelines on surgical procedures: an interpretation," Hepatobiliary Surgery and Nutrition, vol. 2, no. 4, pp. 234-235, 2013.

[2] S. Farkas, C. Hackl, and H. J. Schlitt, "Overview of the indications and contraindications for liver transplantation," Cold Spring Harbor Perspectives in Medicine, vol. 4, no. 5, Article ID a015602, 2014.

[3] E. Tartour, H. Pere, B. Maillere et al., "Angiogenesis and immunity: a bidirectional link potentially relevant for the monitoring of antiangiogenic therapy and the development of novel therapeutic combination with immunotherapy," Cancer and Metastasis Reviews, vol. 30, no. 1, pp. 83-95, 2011.

[4] M. Palmowski, J. Huppert, G. Ladewig et al., "Molecular profiling of angiogenesis with targeted ultrasound imaging: early assessment of antiangiogenic therapy effects," Molecular Cancer Therapeutics, vol. 7, no. 1, pp. 101-109, 2008.

[5] K. Jiang, W.-Z. Zhang, Y. Liu et al., “"One-off” complete radiofrequency ablation for hepatocellular carcinoma in a "high-risk location" adjacent to the major bile duct and hepatic blood vessel," Cell Biochemistry and Biophysics, vol. 69, no. 3, pp. 605617, 2014.

[6] R. T.-P. Poon, I. O.-L. Ng, C. Lau et al., “Tumor microvessel density as a predictor of recurrence after resection of hepatocellular carcinoma: a prospective study," Journal of Clinical Oncology, vol. 20, no. 7, pp. 1775-1785, 2002.

[7] "Oral anti-angiogenesis treatment plus chemotherapy is not more efficacious than bevacizumab plus chemotherapy in metastatic colorectal cancer," Oncology (Williston Park), vol. 25, no. 12, p. 1232, 2011.

[8] S. M. Lee, P. Baas, and H. Wakelee, "Anti-angiogenesis drugs in lung cancer," Respirology, vol. 15, no. 3, pp. 387-392, 2010.

[9] C.-Y. Sang, X.-H. Xu, W.-W. Qin, J.-F. Liu, L. Hui, and S.W. Chen, "DPMA, a deoxypodophyllotoxin derivative, induces apoptosis and anti-angiogenesis in non-small cell lung cancer A549 cells," Bioorganic \& Medicinal Chemistry Letters, vol. 23, no. 24, pp. 6650-6655, 2013.

[10] T. M. B. Nguyen, I. V. Subramanian, X. Xiao et al., "Endostatin induces autophagy in endothelial cells by modulating Beclin 1 and $\beta$-catenin levels," Journal of Cellular and Molecular Medicine, vol. 13, no. 9 B, pp. 3687-3698, 2009.
[11] Y.-P. Chau, S.-Y. Lin, J. H.-C. Chen, and M.-H. Tai, "Endostatin induces autophagic cell death in EAhy926 human endothelial cells," Histology and Histopathology, vol. 18, no. 3, pp. 715-726, 2003.

[12] H. Wang, F. Wei, H. Li, X. Ji, S. Li, and X. Chen, "Combination of oncolytic adenovirus and endostatin inhibits human retinoblastoma in an in vivo mouse model," International Journal of Molecular Medicine, vol. 31, no. 2, pp. 377-385, 2013.

[13] H.-F. Song, X.-W. Liu, H.-N. Zhang et al., "Pharmacokinetics of His-tag recombinant human endostatin in Rhesus monkeys," Acta Pharmacologica Sinica, vol. 26, no. 1, pp. 124-128, 2005.

[14] Y. Ling, Y. Yang, N. Lu et al., "Endostar, a novel recombinant human endostatin, exerts antiangiogenic effect via blocking VEGF-induced tyrosine phosphorylation of KDR/Flk-1 of endothelial cells," Biochemical and Biophysical Research Communications, vol. 361, no. 1, pp. 79-84, 2007.

[15] H. Kim, M.-S. Park, Y. N. Park et al., "Preoperative radiologic and postoperative pathologic risk factors for early intra-hepatic recurrence in hepatocellular carcinoma patients who underwent curative resection," Yonsei Medical Journal, vol. 50, no. 6, pp. 789-795, 2009.

[16] B. K. Sharma, R. Srinivasan, S. Kapil et al., "Angiogenic and antiAngiogenic factor gene transcript level quantitation by quantitative real time PCR in patients with hepatocellular carcinoma," Molecular Biology Reports, vol. 40, no. 10, pp. 5843-5852, 2013.

[17] N. Li, Z.-L. Jin, Z.-J. Liu, J. Wang, and K. Li, “Efficacy of endostar combined with chemotherapy in multi-cycle treatment of patients with advanced non-small cell lung cancer," Zhonghua Zhong Liu Za Zhi, vol. 33, no. 12, pp. 937-942, 2011.

[18] K. Javaherian, T.-Y. Lee, R. M. T. T. Sjin, G. E. Parris, and L. Hlatky, "Two endogenous antiangiogenic inhibitors, endostatin and angiostatin, demonstrate biphasic curves in their antitumor profiles," Dose-Response, vol. 9, no. 3, pp. 369-376, 2011.

[19] A. Moroz, F. K. Delella, R. Almeida et al., "Finasteride inhibits human prostate cancer cell invasion through MMP2 and MMP9 downregulation," PLoS ONE, vol. 8, no. 12, Article ID e84757, 2013.

[20] Y. Zeng, K. Opeskin, J. Goad, and E. D. Williams, “Tumorinduced activation of lymphatic endothelial cells via vascular endothelial growth factor receptor-2 is critical for prostate cancer lymphatic metastasis," Cancer Research, vol. 66, no. 19, pp. 9566-9575, 2006.

[21] D.-I. Sun, I. T. Nizamutdinova, Y. M. Kim et al., "Bisacurone inhibits adhesion of inflammatory monocytes or cancer cells to endothelial cells through down-regulation of VCAM-1 expression," International Immunopharmacology, vol. 8, no. 9, pp. 1272-1281, 2008.

[22] S. Ghosh, M. B. Joshi, D. Ivanov et al., "Use of multicellular tumor spheroids to dissect endothelial cell-tumor cell interactions: a role for T-cadherin in tumor angiogenesis," FEBS Letters, vol. 581, no. 23, pp. 4523-4528, 2007.

[23] P. Vader, R. van der Meel, M. H. Symons et al., "Examining the role of Racl in tumor angiogenesis and growth: a clinically relevant RNAi-mediated approach," Angiogenesis, vol. 14, no. 4, pp. 457-466, 2011.

[24] M. G. Garcia, J. Bayo, M. F. Bolontrade et al., "Hepatocellular carcinoma cells and their fibrotic microenvironment modulate bone marrow-derived mesenchymal stromal cell migration in vitro and in vivo," Molecular Pharmaceutics, vol. 8, no. 5, pp. 1538-1548, 2011. 
[25] A. Kolmakova, M. Rajesh, D. Zang, R. Pili, and S. Chatterjee, "VEGF recruits lactosylceramide to induce endothelial cell adhesion molecule expression and angiogenesis in vitro and in vivo," Glycoconjugate Journal, vol. 26, no. 5, pp. 547-558, 2009.

[26] J. E. Holley, J. Newcombe, J. L. Whatmore, and N. J. Gutowski, "Increased blood vessel density and endothelial cell proliferation in multiple sclerosis cerebral white matter," Neuroscience Letters, vol. 470, no. 1, pp. 65-70, 2010.

[27] S. Saraswati and S. S. Agarwal, "Strychnine inhibits inflammatory angiogenesis in mice via down regulation of VEGF, TNF- $\alpha$ and TGF- $\beta$," Microvascular Research, vol. 87, pp. 7-13, 2013.

[28] K. Bentley, C. A. Franco, A. Philippides et al., "The role of differential VE-cadherin dynamics in cell rearrangement during angiogenesis," Nature Cell Biology, vol. 16, no. 4, pp. 309-321, 2014.

[29] X.-N. Ma, Q.-P. Li, and Z.-C. Feng, "Research progress in cytokines and signaling pathways for promoting pulmonary angiogenesis and vascular development," Zhongguo Dang Dai Er Ke Za Zhi, vol. 15, no. 9, pp. 800-805, 2013. 


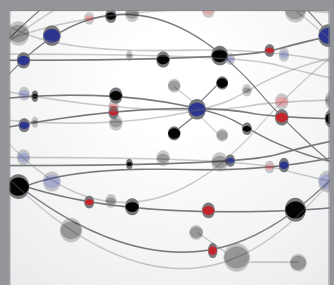

The Scientific World Journal
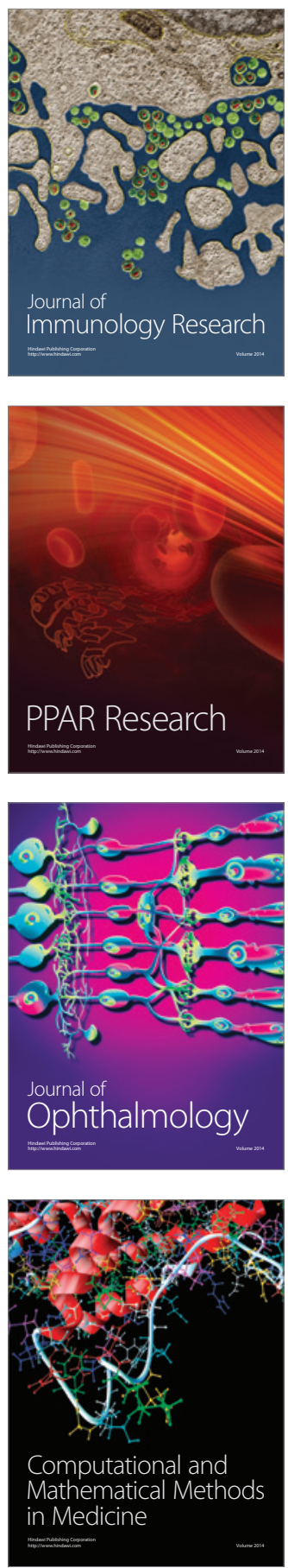

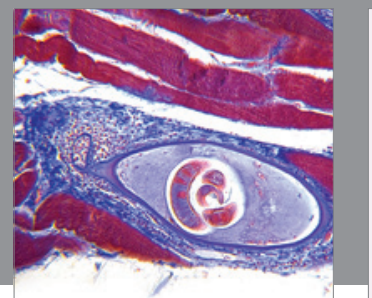

Gastroenterology

Research and Practice
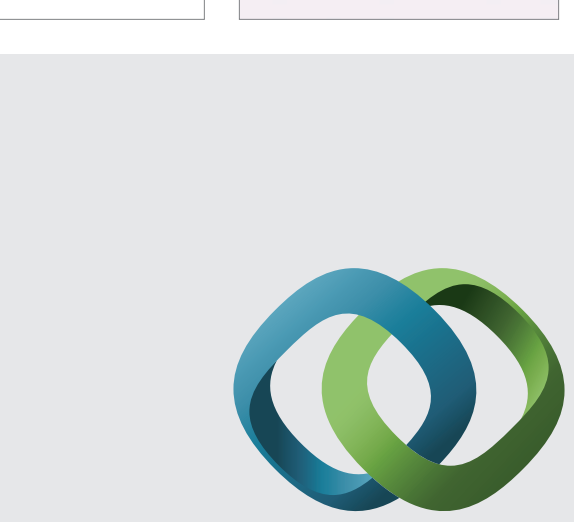

\section{Hindawi}

Submit your manuscripts at

http://www.hindawi.com
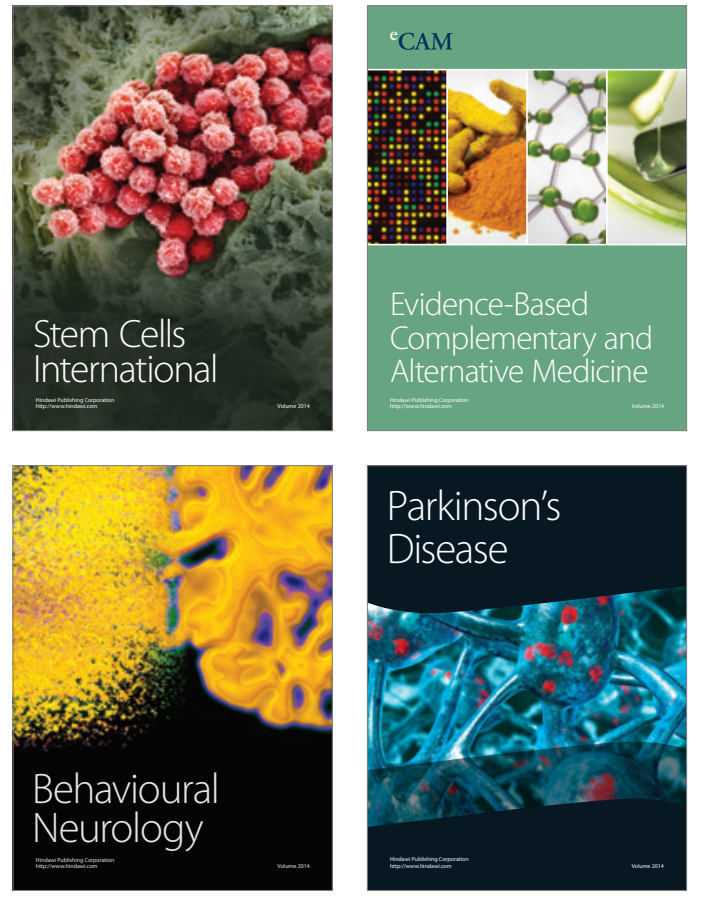
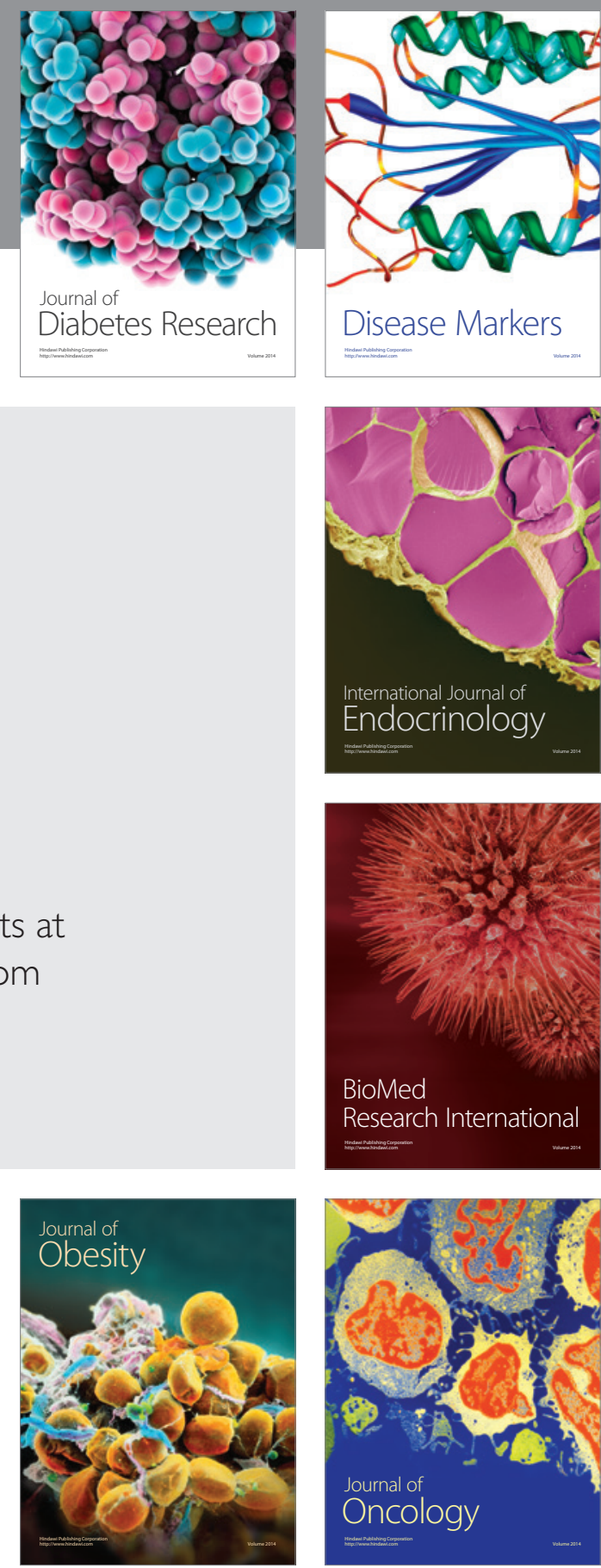

Disease Markers
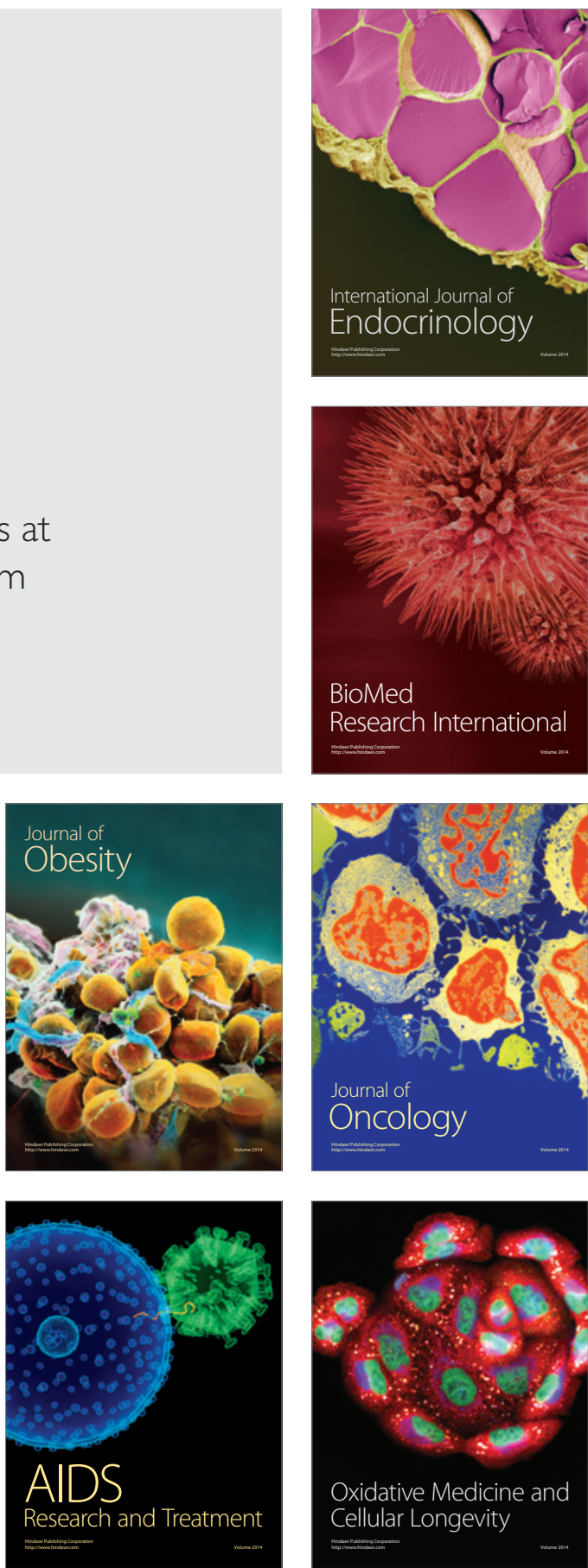